\title{
Monocapas de ácidos grasos. II. Ácido esteárico sobre disoluciones acuosas que contienen solutos con grupos funcionales alcohólicos.
}

\author{
Por J.M. Rodríguez Patino, J. de la Fuente Feria y C. Gómez Herrera. \\ Departamento de Ingeniería Química. Facultad de Química. \\ Universidad de Sevilla, C/. Prof. García González, s/n. \\ 41012 - SEVILLA (España). Fax: (95) 4624952
}

\section{RESUMEN}

Monocapas de ácidos grasos. II. Acido esteárico sobre disoluciones acuosas que contienen solutos con grupos funcionales alcohólicos.

Se ha estudiado la estructura y estabilidad de monocapas de ácido esteárico esparcidas sobre disoluciones acuosas que contienen glicerina, glucosa y sacarosa. Las experiencias se han realizado en una balanza de superficie comercial tipo Langmuir y se ha operado en condiciones isotérmicas.

Las monocapas presentan un polimorfismo estructural que depende de la composición del medio acuoso y de la temperatura. La estabilidad de la monocapa está muy relacionada con la estructura que adopta sus moléculas. Las monocapas con estructuras más expandidas suelen poseer mayor estabilidad.

PALABRAS-CLAVE: Acido esteárico - Balanza de superficie Emulsificante - Monocapa.

\section{SUMMARY}

Monolayers of fatty acids. II. Stearic acid on aqueous solutions containing solutes with alcoholic functional groups.

The structure and the stability of stearic acid monolayers spread on aqueous solutions with glycerol, glucose or sucrose are studied using a commercial film balance (Lauda). The experiments were carried out isothermically.

Monolayers exhibit structural polymorphism depending on temperature and subphase composition. The stability of the molecules on the subphase is a function of their structure. Generally, the most stable monolayers are those with more expanded structures. balance.

KEY-WORDS: Emulsifier - Monolayer - Stearic acid - Surface

\section{1.-INTRODUCCIÓN}

Entre las características de las monocapas de los aditivos alimentarios utilizados como emulsionantes y estabilizadores de interfases, de interés en el procesado de emulsiones y de espumas (1-4), caben citar la estructura que adoptan las moléculas que las constituyen y su estabilidad. La estructura de la monocapa es de interés por su incidencia sobre las propiedades superficiales de la película, tales como viscosidad, elasticidad y compresibilidad (5-7), y sobre la estabilidad de emulsiones y espumas, en cuyas formulaciones se incluyen estas sustancias (8-10). La estabilidad de la monocapa, caracterizada en las aplicaciones alimentarias de estos aditivos, por su solubilidad en la subfase, puede determinar la fracción de aditivo utilizado, cuya cantidad total en la formulación se encuentra regulada legalmente (11), que es útil en las etapas de procesado del alimento o en las propiedades del producto final. Ambas características son muy dependientes de las interacciones que se producen entre las moléculas del emulsionante que forman la monocapa y entre estas y los componentes de la subfase (12). La influencia ejercida por la composición de la subfase puede derivarse del efecto de la misma sobre las propiedades físicas del medio, tales como densidad, viscosidad y tensión superficial $(9,13)$.

Los objetivos del presente trabajo pretenden ampliar los estudios realizados previamente sobre monocapas de ácidos grasos y que han sido publicados en esta revista en un artículo anterior (14). Por su importancia práctica, se han estudiado las características de monocapas de ácido esteárico esparcidas sobre disoluciones de glicerina, glucosa y sacarosa, con distintas concentraciones de soluto y a distintas temperaturas.

\section{2.-MATERIALES Y MÉTODO}

Las isotermas (curvas presión de esparcimiento, $\pi$, frente al área por molécula, A) se han obtenido en una balanza de superficie comercial tipo Langmuir, 
equipada con un sistema de medida continuo y registrador. La temperatura de la bandeja que contiene la monocapa y la subfase se mantiene constante durante cada operación mediante un termostato, con una sensibilidad en el control de temperatura de \pm $0^{\prime} 01-0^{\prime} 03^{\circ} \mathrm{C}$. La forma de operar se ha descrito en un trabajo previo (14).

Los productos usados son de calidad analítica y no han sido sometidos a purificación posterior: ácido esteárico (Merck, Cat. 971), glicerina (Merck, Cat. 4093), glucosa (Merck, Cat. 8346), sacarosa (Merck, Cat. 7654). El agua fue purificada mediante una unidad de filtración Millipore (Mille $Q^{T M}$ ). Los lípidos fueron esparcidos sobre la subfase previa disolución en benceno (Merck, Cat. 1783). La falta de actividad superficial de los componentes de la subfase y del benceno usado se ha comprobado experimentalmente.

\section{3.- RESULTADOS Y DISCUSIÓN}

3.1. Ácido esteárico esparcido sobre disoluciones de glicerina en la subfase.

La influencia de la concentración de glicerina en la subfase se estudia a partir de los resultados obtenidos tras esparcir el ácido esteárico sobre disoluciones con concentraciones en soluto comprendidas entre 0 '2 $\mathrm{mol} / \mathrm{L}$ y 0 '5 $\mathrm{mol} / \mathrm{L}$.

\section{Disolución acuosa a 0'2 mol/L de glicerina como subfase.}

Los resultados obtenidos al esparcir el ácido esteárico sobre la disolución acuosa de glicerina de concentración 0'2 mol/L se muestran en la figura 1. En la zona de estudio correspondiente al intervalo de temperaturas comprendido entre $20^{\circ} \mathrm{C}$ y $40^{\circ} \mathrm{C}$, la monocapa posee una estructura de líquido condensado, con un área límite de 0 '167 $\mathrm{nm}^{2} /$ molécula, que es independiente del valor de la temperatura. El bajo valor del área límite, así como la independencia entre las isotermas registradas y la temperatura, en el intervalo indicado, son dos características del sistema que pueden atribuirse a la existencia de disolución de moléculas de la monocapa en la subfase (14). La disolución de la monocapa es máxima a la temperatura de $45^{\circ} \mathrm{C}$. En efecto, las características de la isoterma registrada a esta temperatura se corresponden con las de una estructura de la monocapa de líquido condensado, aunque con una solicitud de área menor que las obtenidas a temperaturas inferiores.

A las temperaturas de $50{ }^{\circ} \mathrm{C}$ y superiores la monocapa ha experimentado una transformación hacia la estructura de líquido expandido. La zona corres-

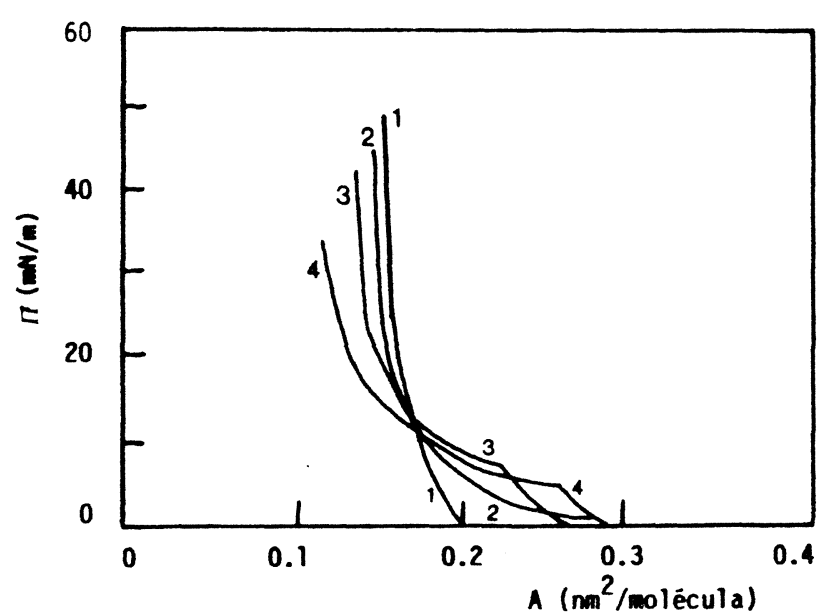

Figura 1

Isotermas de compresión presión superficial-área por molécula de ácido esteárico.

Subfase: disolución acuosa de glicerina a $0.2 \mathrm{~mol} / \mathrm{L}$. Temperaturas. 1: $20-40^{\circ} \mathrm{C} ; 2: 45^{\circ} \mathrm{C} ; 3: 50^{\circ} \mathrm{C} ; 4: 57.5^{\circ} \mathrm{C}$.

pondiente a la de existencia de la meseta característica de dicha estructura se incrementa con la temperatura (véase que la presión a la cual comienza el registro de la meseta disminuye al aumentar la temperatura). Es decir, la estructura de la monocapa se hace más expandida al aumentar la temperatura y, para un valor de la variable de $57^{\prime} 5^{\circ} \mathrm{C}$, es prácticamente gaseosa. La solubilidad de la monocapa se potencia a estas temperaturas pues, aunque la estructura de la misma es más expandida, sus registros aparecen a menores solicitudes de área por las moléculas, al aumentar la temperatura del sistema.

Finalmente, la presión de colapso de estas monocapas disminuye al aumentar la temperatura, según las relaciones establecidas en la tabla I. Es decir, al aumentar la temperatura, la reducción de las interacciones entre las cadenas hidrocarbonadas del ácido esteárico, que se corresponde con una disminución de la presión de colapso, es coincidente con la adopción de una estructura más expandida por parte de sus moléculas e, incluso, puede incidir sobre la importancia relativa de las interacciones entre dichas moléculas y los constituyentes de la subfase, que se manifestaría por la existencia de una desestabilización por disolución de la monocapa $(15,16)$.

\section{Disolución acuosa a 0’3 mol/L de glicerina como subfase.}

Las isotermas correspondientes a estas experiencias se representan en la figura 2. En el intervalo comprendido entre 5 y $35^{\circ} \mathrm{C}$ la forma y localización de las isotermas son independientes de la temperatura. En dicho intervalo la monocapa posee una estructura de líquido condensado, con un valor del 
Tabla 1

Presión de colapso, $\pi_{c}$, de monocapas de ácido esteárico esparcidas sobre disoluciones de glicerina a $0,2 \mathrm{~mol} / \mathrm{L}$.

$\begin{array}{lllllll}T(\Omega C) & 20 & 30 & 40 & 45 & 50 & 57.5 \\ \pi_{C}(\mathrm{mN} / \mathrm{m}) & 46.9 & 46.5 & 45.9 & 45.0 & 47.2 & 35.0\end{array}$

área límite de 0 '178 $\mathrm{nm}^{2} /$ molécula, y es parcialmente soluble en la subfase. A la temperatura de $40^{\circ} \mathrm{C}$ la monocapa mantiene la misma estructura que a temperaturas inferiores, aunque se hace más expandida: el valor deducido para el área límite es de 0'182 $\mathrm{nm}^{2} /$ molécula.

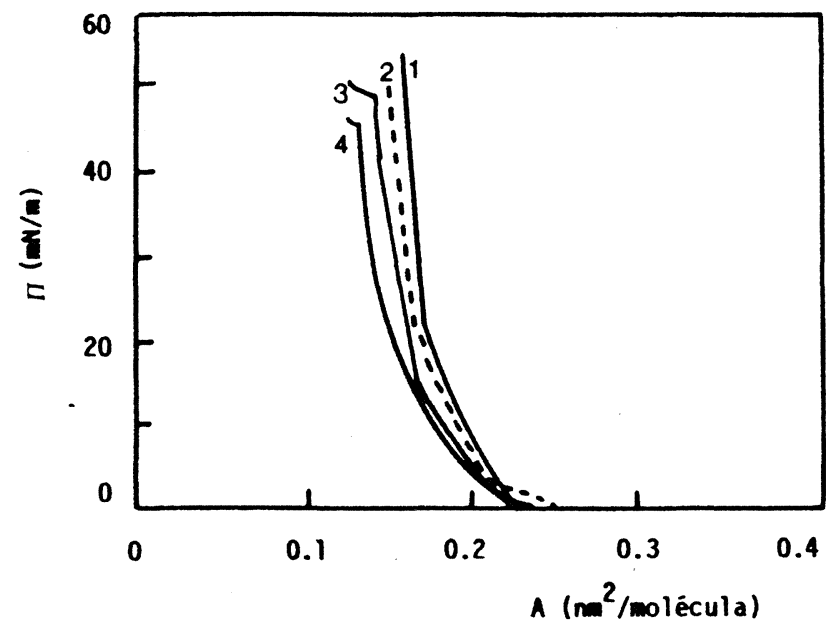

Figura 2

Isotermas de compresión presión superficial-área por molécula de ácido esteárico.

Subfase: disolución acuosa de glicerina a $0.3 \mathrm{~mol} / \mathrm{L}$.

Temperaturas. 1: $5-35^{\circ} \mathrm{C} ; 2: 40^{\circ} \mathrm{C} ; 3: 46^{\circ} \mathrm{C}$ (primera compresión); 4: $46^{\circ} \mathrm{C}$ (segunda compresión).

Los desplazamientos de las isotermas registradas tras compresiones sucesivas de la monocapa a la temperatura de $46{ }^{\circ} \mathrm{C}$ son indicativos de un proceso de relajación hacia el equilibrio después de producirse una pérdida de ácido esteárico desde la monocapa, bien por disolución del mismo en la subfase o bien por su arrastre hacia el aire por evaporación de la misma a estos valores de la variable. La forma de la isoterma puede atribuirse a la existencia de una estructura gaseosa en la monocapa.

\section{Disoluse.}

Las características fundamentales de las monocapas de ácido esteárico esparcidas sobre una disolución acuosa de glicerina de concentración 0 '5 mol/L en soluto, deducidas de los resultados representados en la figura 3 , correspondientes al intervalo de temperaturas estudiadas, entre 5 y $40{ }^{\circ} \mathrm{C}$, se pueden resumir en dos puntos:

* Adopción por las moléculas de la monocapa de una estructura gaseosa que se hace más expandida al aumentar la temperatura.

* Existencia de disolución de la monocapa que se potencia al incrementarse la temperatura: las isotermas se registran a menores áreas por molécula al aumentar la temperatura, aunque su estructura se expanda en este mismo sentido.

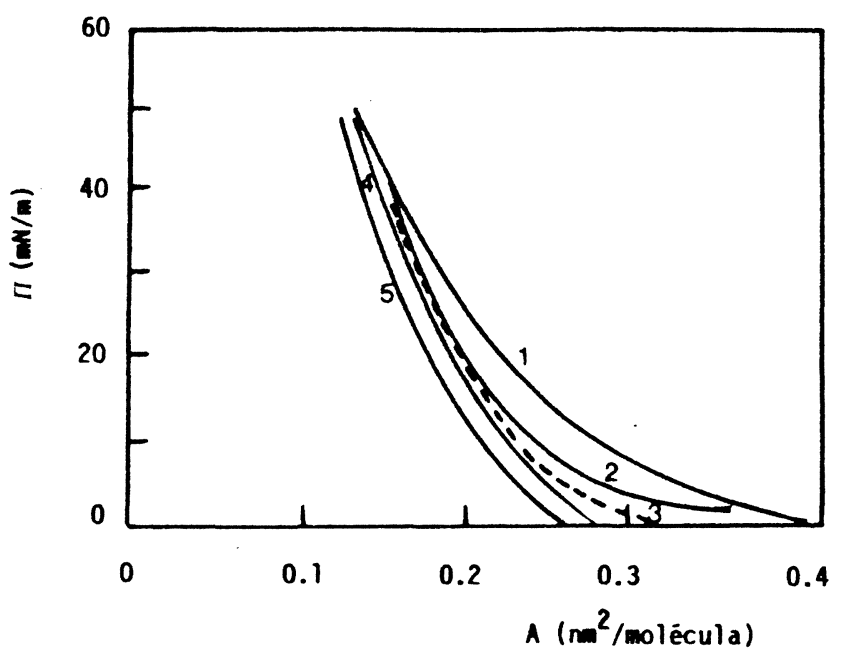

Figura 3

Isotermas de compresión presión superficial-área por molécula de ácido esteárico.

Subfase: disolución acuosa de glicerina a $0.5 \mathrm{~mol} / \mathrm{L}$.

Temperaturas. $1: 5^{\circ} \mathrm{C} ; 2: 10-25^{\circ} \mathrm{C} ; 3: 30^{\circ} \mathrm{C} ; 4: 35^{\circ} \mathrm{C} ; 5$ : $40^{\circ} \mathrm{C}$

\section{Discusión global de resultados con glicerina como subfase.}

Existe una interdependencia entre las influencias de la concentración de glicerina y de la temperatura sobre las características de las monocapas de ácido esteárico que se puede concretar en lo siguiente:

* Cualquiera que sea la temperatura del sistema, un aumento en la concentración de glicerina en la subfase se traduce en una expansión de la estructura de la monocapa. A título de ejemplo, en la figura 4 se representa el efecto comentado a la temperatura de $20^{\circ} \mathrm{C}$. Un efecto similar se observa al aumentar la temperatura, manteniéndose constante la concentración de glicerina en la subfase. 


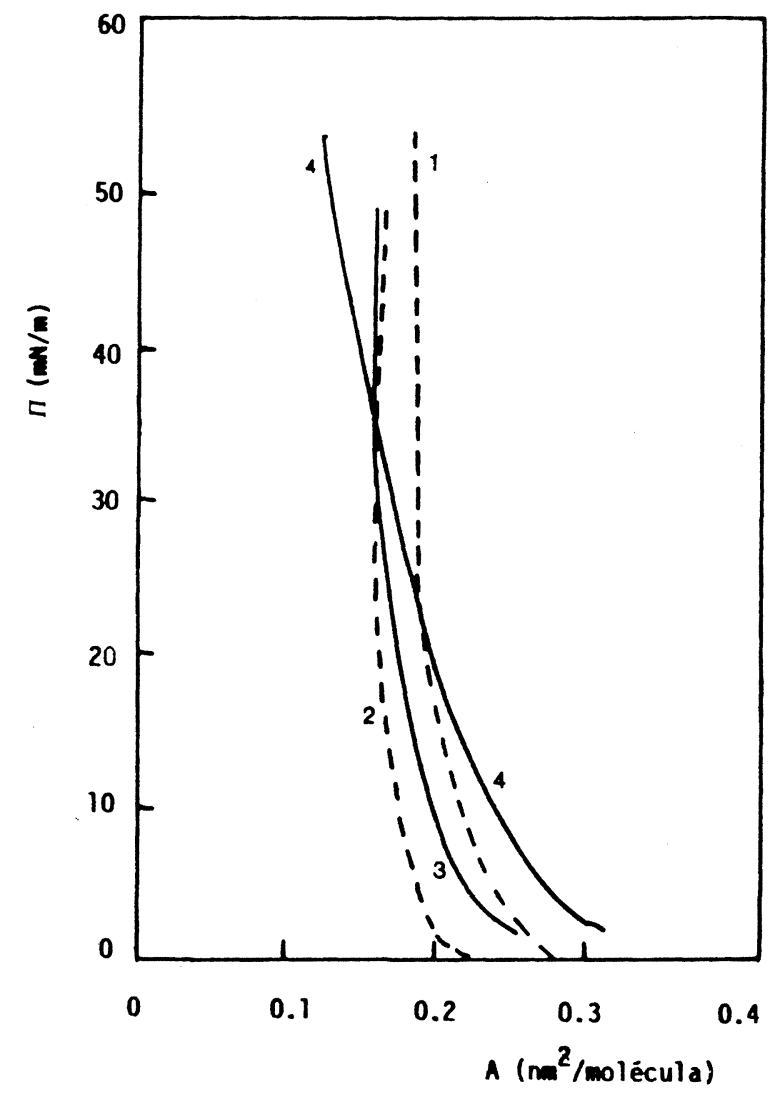

Figura 4

Isotermas de compresión presión superficial-área por molécula de ácido esteárico a $20^{\circ} \mathrm{C}$.

Subfase: disolución acuosa de glicerina, $1: 0 \mathrm{~mol} / \mathrm{L} ; 2$ : $0.2 \mathrm{~mol} / \mathrm{L} ; 3: 0.3 \mathrm{~mol} / \mathrm{L} ; 4: 0.5 \mathrm{~mol} / \mathrm{L}$.

* Según sea la temperatura del sistema o la concentración de glicerina en la subfase, las monocapas de ácido esteárico presentan un polimorfismo adoptando estructuras de sólido, líquido condensado o líquido expandido, observándose transformaciones en este sentido al aumentar los valores de las citadas variables.

* La solubilidad de la monocapa es sensible a los cambios de temperatura y de concentración de glicerina en la subfase. No obstante, los aumentos de los valores de las citadas variables no afectan con el mismo nivel de significación a la solicitud de área molecular.

\section{2 Ácido esteárico esparcido sobre disolu-} ciones de glucosa en la subfase.

Se han estudiado las características de las monocapas de ácido esteárico esparcido sobre disoluciones acuosas de glucosa con tres concentraciones distintas. A saber: 0'05 mol/L, 0'2 mol/L y 0'5 mol/ L.

\section{Disolución acuosa de glucosa a 0’05 mol/L como subfase.}

Las isotermas correspondientes a las monocapas esparcidas sobre la citada subfase se representan en la figura 5 . De los resultados obtenidos se pueden deducir como características fundamentales de las monocapas en función de la temperatura las siguientes:

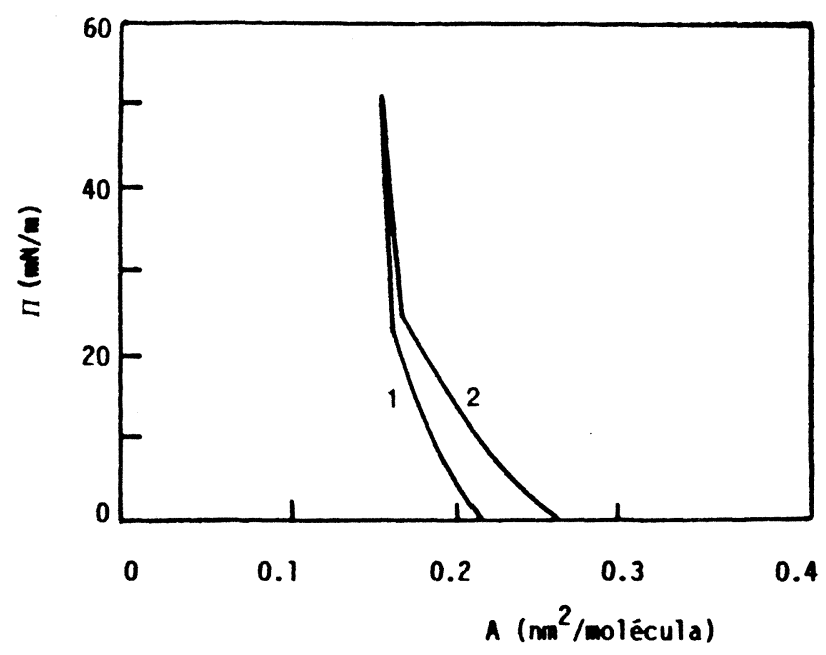

Figura 5

Isotermas de compresión presión superficial-área por molécula de ácido esteárico.

Subfase: disolución acuosa de glucosa a $0.05 \mathrm{~mol} / \mathrm{L}$. Temperaturas. $1: 5$, y $10^{\circ} \mathrm{C} ; 2: 15-45^{\circ} \mathrm{C}$.

* A temperaturas de $5{ }^{\circ} \mathrm{C}$ a $10^{\circ} \mathrm{C}$ la monocapa posee una estructura de líquido condensado, con un valor del área límite de 0'174 $\mathrm{nm}^{2} /$ molécula y una presión característica a la cual se produce la transición entre las estructuras líquido condensada y sólida $\left(\pi^{*}\right)$ de $23^{\prime} 6 \mathrm{mN} / \mathrm{m}$.

* En el intervalo de temperaturas comprendido entre $15^{\circ} \mathrm{C}$ y $45^{\circ} \mathrm{C}$ las moléculas de la monocapa adquieren una estructura de líquido expandido, si bien, al igual que ocurre en el intervalo anterior de temperaturas, los valores del área límite y de la presión características son de 0'186 $\mathrm{nm}^{2} /$ molécula y $24,5 \mathrm{mN}$ / $\mathrm{m}^{2}$, respectivamente, independientemente del valor de la temperatura.

* La disminución del área límite y la expansión de la estructura de la monocapa, con el aumento de temperatura, así como su independencia de las solicitudes de área por molécula, dentro del intervalo de temperatura de $5^{\circ} \mathrm{C}$ a $45^{\circ} \mathrm{C}$ confirman la existencia de solubilidad de la monocapa en la subfase.

Disolución acuosa de glucosa a 0’2 $\mathrm{mol} / \mathrm{L}$ como subfase.

Las experiencias realizadas con este sistema se representan en la figura 6 . Como características 
fundamentales de estas monocapas se pueden destacar las siguientes:

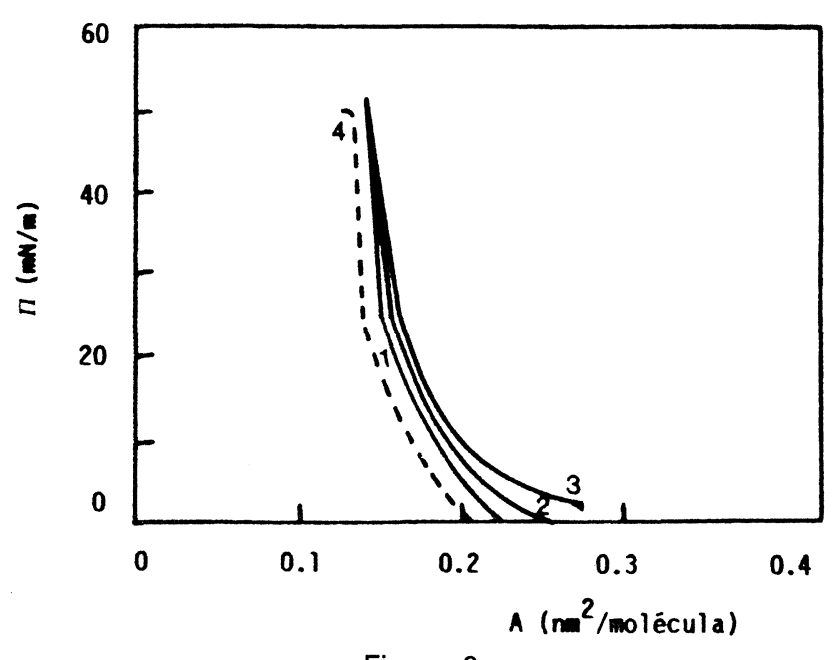

Figura 6

Isotermas de compresión presión superficial-área por molécula de ácido esteárico.

Subfase: disolución acuosa de glucosa a $0.2 \mathrm{~mol} / \mathrm{L}$. Temperaturas. 1: $5^{\circ} \mathrm{C} ; 2: 20^{\circ} \mathrm{C} ; 3: 25^{\circ} \mathrm{C}$.

Subfase: disolución acuosa de glucosa a $0.5 \mathrm{~mol} / \mathrm{L}$. Temperaturas. $4: 5-40^{\circ} \mathrm{C}$.

* Las moléculas de la monocapa adoptan una estructura líquido condensada que es independiente de la temperatura del sistema.

La presión de colapso es de $51^{\prime} 1 \mathrm{mN} / \mathrm{m}$ aproximadamente.

* Al aumentar la temperatura se produce una expansión de la estructura que se manifiesta en un aumento del área límite y en un incremento de la presión característica, según se expone en la tabla II.
* No se puede apreciar el efecto de la temperatura sobre la estabilidad de la monocapa, aunque es posible que la citada variable posibilite la disolución de ácido esteárico en la subfase, pues la expansión que experimenta la monocapa al aumentar la temperatura no se refleja en incrementos en la solicitud de área por las moléculas que la compone, como ocurre con las experiencias realizadas utilizando menor concentración de soluto en la subfase estudiada en el apartado anterior.

\section{Disolución acuosa de glucosa a 0'5 mol/L como subfase.}

La característica fundamental de las isotermas registradas al esparcir ácido esteárico sobre disolución acuosa de concentración 0'5 mol/L en glucosa es su independencia de la temperatura, según se muestra en la curva 4 que se incluye en la figura 6 . La estructura adoptada por las moléculas es de líquido condensado con un valor de la presión característica de $23^{\prime} 4 \mathrm{mN} /$ $\mathrm{m}$ y un área límite de 0'152 $\mathrm{nm}^{2} /$ molécula. Este valor del área límite así como la independencia entre la solicitud de área por las moléculas y la temperatura ha de atribuirse, por las razones expuestas en apartados previos, a la disolución de las moléculas de la monocapa en las condiciones de experimentación.

\section{Discusión global de resultados con glucosa como subfase.}

Esta discusión se centra en la influencia de la concentración de glucosa en la subfase, y su interdependencia con la temperatura del sistema, sobre

Tabla II

Presión característica y área límite de monocapas de ácido esteárico esparcidas sobre disolución acuosa de glucosa a $0,2 \mathrm{~mol} / \mathrm{L}$.

$\begin{array}{lcll}T(8 \mathrm{C}) & 5 & 20 & 25 \\ \pi^{*}(\mathrm{mN} / \mathrm{m}) & 23^{\prime} 6 & 25^{\prime} 8 & 28^{\prime} 5 \\ A_{11 \mathrm{~m}}\left(\mathrm{~nm}^{2} / \mathrm{mol \delta c}\right) & 0^{\prime} 170 & 0^{\prime} 168 & 0.166\end{array}$

las características de monocapas esparcidas de ácido esteárico.

La concentración de glucosa en la subfase incide sobre la estructura de la monocapa produciendo una contracción de la misma desde una estructura de líquido expandido, a bajas concentraciones de soluto, a una estructura de líquido condensado, a las concentraciones más elevadas de glucosa. Un ejemplo de la citada dependencia son los resultados representados en la figura 7 , referidos a la temperatura de $20^{\circ} \mathrm{C}$. Las áreas límites que se deducen de estas isotermas se exponen en la tabla III.

Estos resultados admiten la siguiente explicación: las interacciones entre las moléculas de la monocapa y las de la disolución pueden traducirse en una atracción de aquellas hacia el interior de la subfase, que se facilitaría por la presencia de las moléculas de glucosa. Al sumergirse la cabeza polar del ácido graso también lo hace parcialmente sus cadenas hidrocarbonadas y el área ocupado por las moléculas 
Tabla III

Areas límite de monocapas de ácido esteárico esparcidas sobre disoluciones acuosas de glucosa $\left(20^{\circ} \mathrm{C}\right)$

$\begin{array}{lccccc}\text { Concentrac18n de } & 0 & 0.05 & 0.1 & 0.2 & 0.5 \\ \text { 8lucosa (mol/L) } & & & & & \\ A_{11 \mathrm{~m}}\left(\mathrm{~nm}^{2} / \mathrm{mol} \text { 8c) }\right. & 0.205 & 0.186 & 0.172 & 0.166 & 0.162\end{array}$

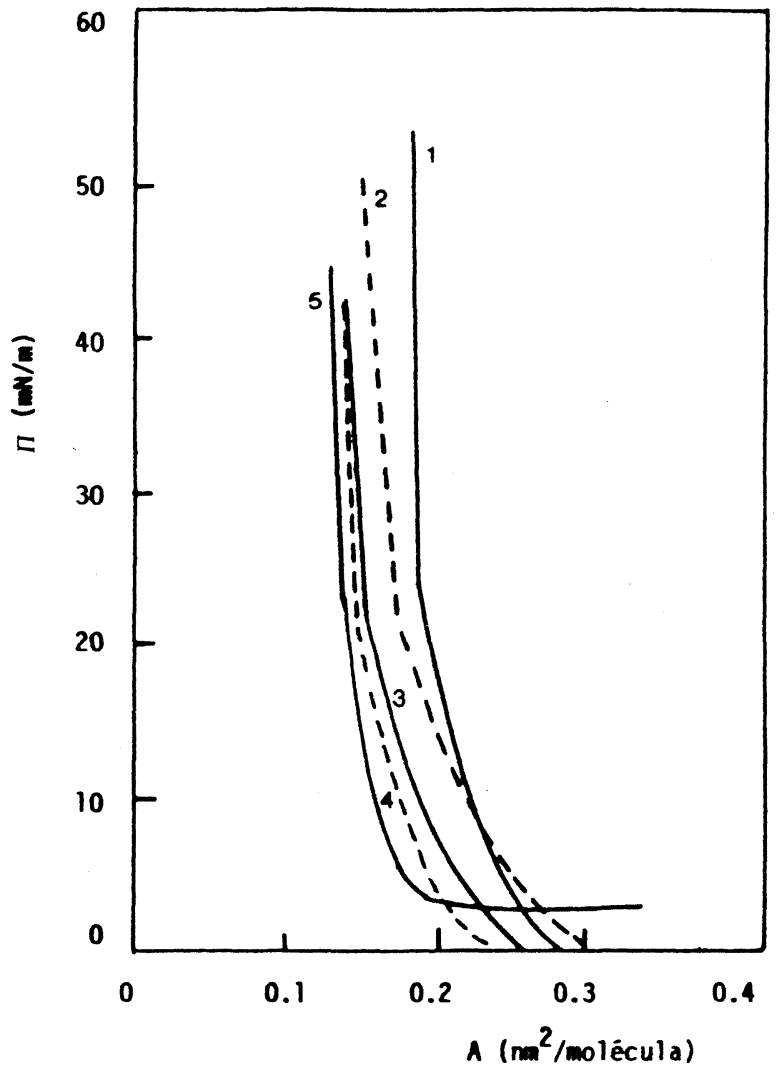

Figura 7

Isotermas de compresión presión superficial-área por molécula de ácido esteárico a $20^{\circ} \mathrm{C}$.

Subfase: disolución acuosa de glicerina. 1: $0 \mathrm{~mol} / \mathrm{L} ; 2$ : $0.05 \mathrm{~mol} / \mathrm{L} ; 3: 0.1 \mathrm{~mol} / \mathrm{L} ; 4: 0.2 \mathrm{~mol} / \mathrm{L} ; 5: 0.5 \mathrm{~mol} / \mathrm{L}$.

se reduce (15), lo que es concordante con los datos presentados en la tabla III. Por la misma razón disminuirían las fuerzas de repulsión entre las citadas cadenas lo que explicaría la reducción que se observa en la presión de colapso al aumentar la concentración de glucosa en la subfase, en general $(15,16)$.

Las interacciones entre las moléculas de la monocapa y de la subfase pueden ser tales que se solubilice una fracción de las mismas (17), efecto que se traduciría igualmente en una reducción en el valor del área límite. No obstante, a partir de los resultados obtenidos no se puede establecer una relación clara entre la concentración de glucosa en la subfase y el grado de disolución de la monocapa. La presencia de glucosa en la subfase, a los valores estudiados de sus concentraciones, ha de modificar además la viscosidad de la misma, un efecto que puede incidir sobre la solubilidad de la monocapa y sobre la cinética del proceso $(15,18)$.

3.3. Ácido esteárico esparcido sobre disoluciones de sacarosa en la subfase.

Las experiencias se han realizado esparciendo las moléculas de ácido esteárico sobre disoluciones acuosas de sacarosa como subfases, de concentraciones de 0'05 mol/L, 0'1 mol/L y 0'5 mol/L.

Disolución acuosa de sacarosa a 0'05 mol/L como subfase.

Según se observa en la figura 8 , las isotermas registradas son prácticamente independientes de la temperatura en el intervalo estudiado, comprendido entre 5 y $40^{\circ} \mathrm{C}$. Por lo tanto puede afirmarse que la estructura de las moléculas en la monocapa no se afecta por la temperatura. La estructura adoptada es de líquido condensado con unos valores del área límite

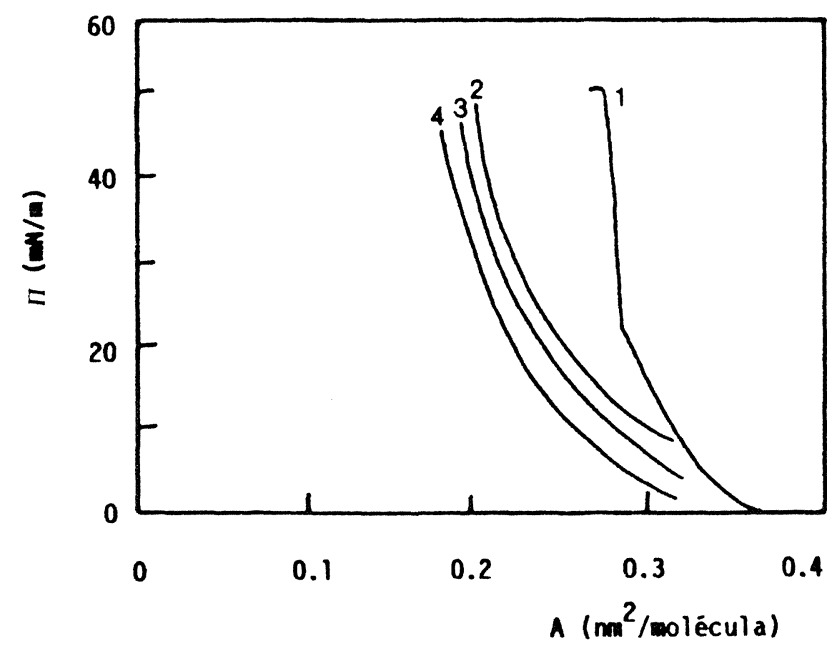

Figura 8

Isotermas de compresión presión superficial-área por molécula de ácido esteárico.

Subfase: disolución acuosa de sacarosa a $0.05 \mathrm{~mol} / \mathrm{L}$. Temperaturas. $1: 5-40^{\circ} \mathrm{C}$.

Subfase: disolución acuosa de sacarosa a $0.1 \mathrm{~mol} / \mathrm{L}$. Temperaturas. 2: $5-20^{\circ} \mathrm{C} ; 3: 25-30^{\circ} \mathrm{C} ; 4: 35-40^{\circ} \mathrm{C}$ 
y de la presión característica de 0’205 $\mathrm{nm}^{2} /$ molécula y 21 ' $5 \mathrm{mN} / \mathrm{m}$, respectivamente. Aunque el valor deducido del área límite es prácticamente coincidente con la distancia entre los átomos de ácido esteárico en su estructura cristalina (19), la constancia en la solicitud de área por las moléculas a cualquier temperatura puede atribuirse a la existencia de disolución de las mismas en la subfase.

\section{Disolución acuosa de sacarosa a 0 '1 mol/L como subfase.}

Las características principales de las monocapas de ácido esteárico esparcidas sobre disolución acuosa de sacarosa de concentración 0'1 mol/L, según se deduce de las isotermas registradas que se incluyen en la figura 8 , se pueden resumir en dos puntos:

* Las moléculas de la monocapa adoptan una estructura gaseosa, incluso a las menores temperaturas. Además, la temperatura no afecta a su estructura pero sí a su expansión, pues las isotermas registradas a las distintas temperaturas son prácticamente paralelas entre sì.

* Las moléculas de la monocapa poseen una solubilidad en la subfase que aumenta al incrementarse la temperatura, según se deduce de los desplazamientos hacia zonas con menores solicitudes de área observados al producirse el citado incremento.

Disolución acuosa de sacarosa a 0'5 mol/L como subfase.

Para estas concentraciones tan elevadas de sacarosa en la subfase las interacciones entre la cabeza polar del ácido esteárico y las moléculas de

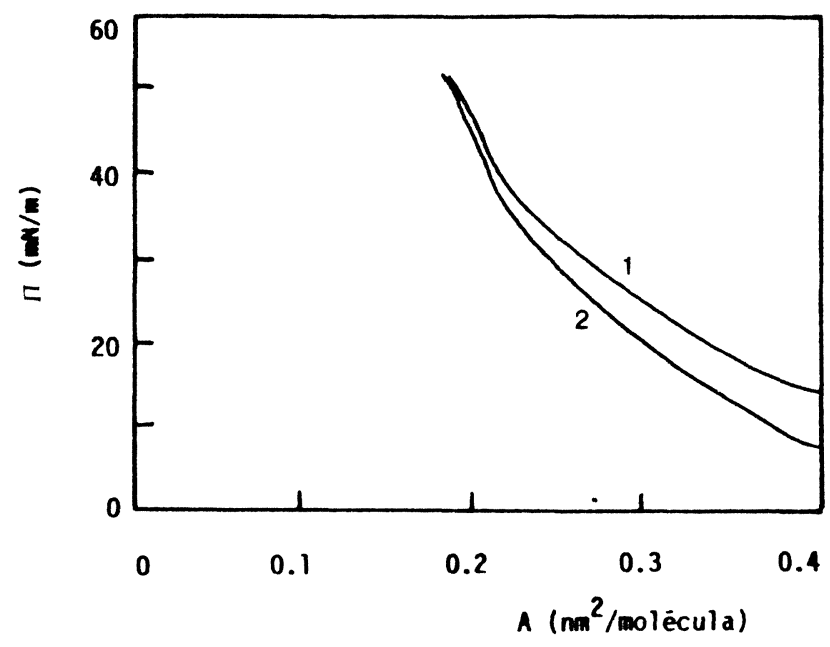

Figura 9

Isotermas de compresión presión superficial-área por molécula de ácido esteárico.

Subfase: disolución acuosa de sacarosa a $0.5 \mathrm{~mol} / \mathrm{L}$.

Temperatura: $20^{\circ} \mathrm{C}$. 1: primera compresión; 2: segunda compresión. la misma en la interfase son tales que se potencia la solubilidad de la monocapa, incluso, a bajas temperaturas. En efecto, según se representa en la figura 9 las isotermas registradas a la temperatura de $20^{\circ} \mathrm{C}$ son concordantes con la adopción por las moléculas de una estructura expandida, prácticamente gaseosa, y se producen desplazamientos hacia menores solicitudes de área al realizarse sobre ellas compresiones sucesivas. Sobre los procesos de relajación antes citados ha de ejercer una influencia decisiva las características reológicas de la subfase (9).

\section{Discusión global de resultados con sacarosa como subfase.}

La concentración de sacarosa en la subfase influye sobre las características de las monocapas de ácido esteárico relacionadas con su estructura y su estabilidad.

De los resultados experimentales obtenidos se deduce la existencia de transiciones de fases en la monocapa, hacia estructuras más expandidas, al aumentar la concentración de sacarosa en la subfase.

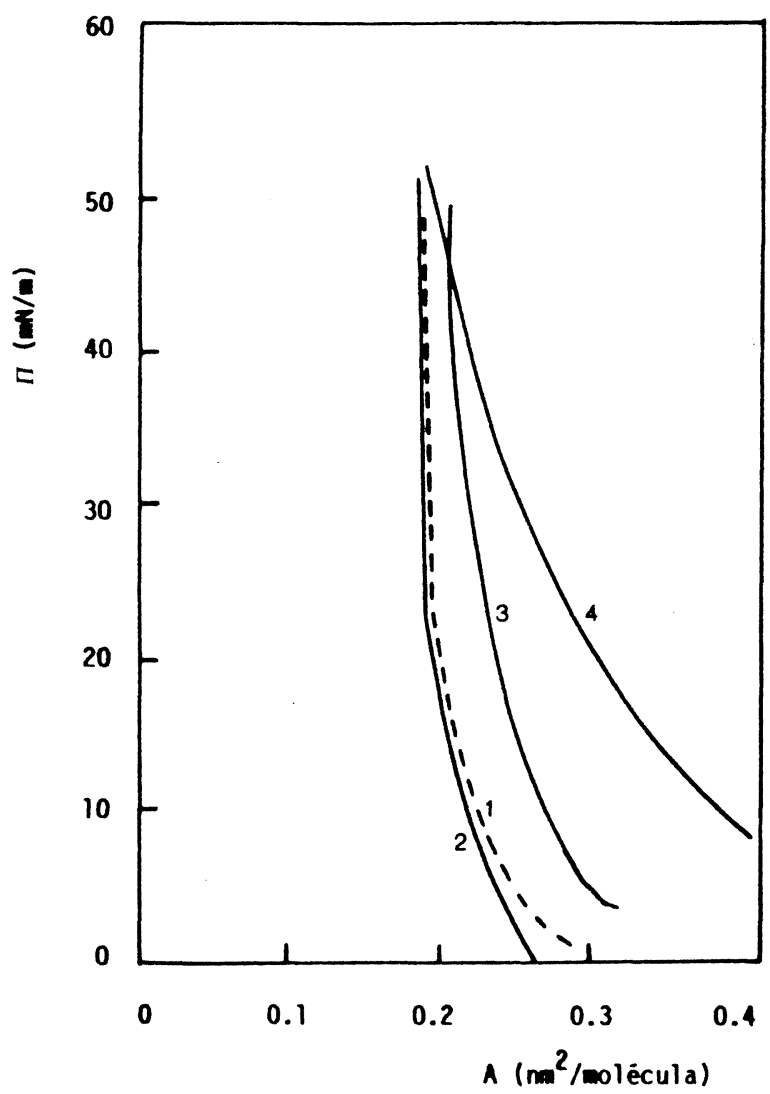

Figura 10

Isotermas de compresión presión superficial-área por molécula de ácido esteárico a $20^{\circ} \mathrm{C}$

Subfases: disoluciones acuosas de sacarosa. 1: $0 \mathrm{~mol} / \mathrm{L}$; 2: $0.05 \mathrm{~mol} / \mathrm{L} ; 3: 0.1 \mathrm{~mol} / \mathrm{L} ; 4: 0.5 \mathrm{~mol} / \mathrm{L}$. 
Concretamente, la estructura de la monocapa pasa de ser líquida condensada a líquida expandida, para transformarse finalmente en gaseosa, cuando la concentración de soluto en la subfase adquiere los valores de 0'05 mol/L, 0'1 mol/L y 0'5 mol/L, respectivamente. A título de ejemplo se representa en la figura 10 la dependencia citada, correspondiente a una temperatura de $20^{\circ} \mathrm{C}$.

Un aumento de la concentración de sacarosa en la subfase induce una desestabilización de la monocapa, incrementando su solubilidad. Esta dependencia es más difícil de establecer a la mayor concentración estudiada (disolución acuosa de sacarosa 0'5 $\mathrm{mol} / \mathrm{L}$ ), por las dificultades experimentales que se introducen al estudiar sistemas con elevada viscosidad en la subfase.

3.4. Comparación entre las distintas subfases estudiadas.

Las monocapas de ácido esteárico poseen una serie de características que son dependientes de la subfase sobre la cual se extienden las moléculas del lípido. Se estudiará en este apartado la influencia de la temperatura y de la concentración de soluto, en función del constituyente utilizado en la disolución acuosa que forma la subfase, sobre las características de las monocapas de ácido esteárico. La discusión se centrará en la incidencias de las variables

Tabla IV

Interdependencia entre la estructura de la monocapa de ácido esteárico y la temperatura, en función de la composición de la subfase.

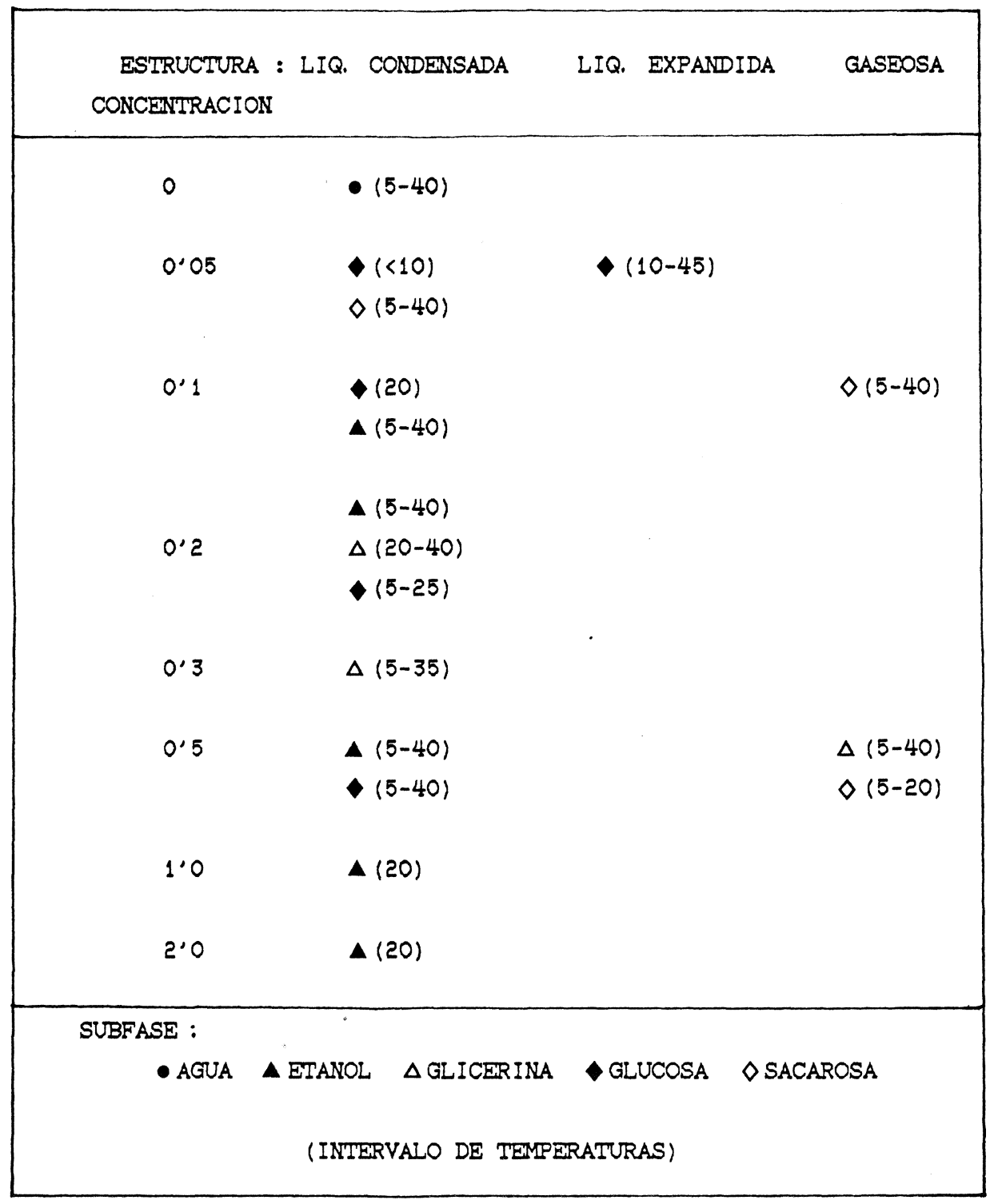


citadas sobre la estructura y la solubilidad de las moléculas de la monocapa. En la citada discusión se incluirán los datos obtenidos con monocapas de ácido esteárico esparcidas sobre agua desionizada y disoluciones de etanol, estudiadas en un artículo anterior (14).

A) Influencia sobre la estructura de la monocapa.

La configuración que adoptan las moléculas que forman la monocapa es función de la temperatura del sistema, independientemente del área disponible para las moléculas. Estas relaciones se resumen en la tabla IV. De la misma se pueden deducir las siguientes consideraciones:

* Un aumento en el número de grupos hidroxilos en la formulación de la molécula de soluto que constituye la disolución de la subfase produce una expansión en la configuración de las moléculas de la monocapa. Es decir, la estructura de la monocapa pasa de ser líquida condensada a ser líquida expandida y finalmente adquiere una configuración gaseosa.

* El efecto del incremento de la concentración de soluto en la subfase se manifiesta mediante una expansión en la estructura de la monocapa.

* Al aumentar la temperatura del sistema se favorece la transición hacia una estructura más expandida de las moléculas que constituyen la monocapa.

* Los efectos antes citados son cualitativamente aditivos, de manera que, en el sistema constituido por una monocapa de ácido esteárico sobre disolución concentrada de sacarosa, la estructura que adoptan las moléculas en la misma es gaseosa, incluso, a los valores inferiores de la temperatura.

* El comportamiento del sistema con etanol como soluto de las disoluciones acuosas que constituyen la subfase es prácticamente diferente de los restantes. Las monocapas de ácido esteárico esparcidas sobre estas disoluciones poseen una configuración líquida condensada a cualquier concentración de soluto y a cualquier temperatura. No obstante, las variables citadas incide sobre la presión característica a la cual se produce la transición LC $\rightleftharpoons S$ (14).

La intervención de la composición de la subfase sobre la estructura de la monocapa puede ser la consecuencia de la influencia que ejercen las propiedades físicas de las disoluciones estudiadas como subfases. La densidad y viscosidad de la subfase puede modificar la estructura de la monocapa, expandiéndola con su aumento hasta que el esfuerzo debido a la tracción viscosa se compense con la presión de esparcimiento de la monocapa $(9,15,20)$. La tensión superficial de la subfase puede intervenir según su valor absoluto, incidiendo sobre la curvatura del menisco formado sobre la superficie de la monocapa comprendida entre las barreras (13). Es posible una intervención de la propiedad según su gradiente, lo que daría lugar al fenómeno conocido como efecto Marangoni $(20,21)$. Cuando las moléculas de la monocapa están en movimiento por la acción del gradiente de tensión superficial, por efecto Marangoni se puede originar un arrastre de líquido de la subfase, lo que repercutiría sobre su estructura. La existencia del efecto Marangoni posee una aplicación práctica en la estabilización de emulsiones y espumas (20).

B) Influencia sobre la solubilidad de la monocapa.

La estabilidad de las monocapas de ácido esteárico, referida en este estudio a la pérdida de moléculas de la película por solubilidad en la subfase, está muy relacionada con su estructura. Se puede afirmar como comportamiento general, según la discusión de resultados realizada previamente, que las estructuras más expandidas tienden a estabilizar a la monocapa, cualquiera que sea el tipo de soluto que forma la disolución utilizada como subfase, o su concentración en la misma.

La temperatura incide siempre de la misma forma sobre la estabilidad de la monocapa: un aumento de temperatura produce un incremento de la solubilidad

Tabla V

Grado de solubilidad de monocapas de ácido esteárico en función de la composición de la subfase.

\begin{tabular}{lll} 
SOLUTO & CONCENTRACION & SOLUBILIDAD \\
\cline { 2 - 3 } & $0 \longrightarrow 0.2$ & Disminuye \\
ETANOL & $0.5 \longrightarrow 2$ & Aumenta \\
ETANOL & $0 \longrightarrow 0.5$ & Disminuye \\
GLICERINA & $0 \longrightarrow 0.5$ & Aumenta \\
GLUCOSA & $0 \longrightarrow 0.1$ & Aumenta \\
SACAROSA & 0 &
\end{tabular}


de las moléculas que constituyen la monocapa, efecto que se incrementa a las temperaturas superiores. Esta influencia de la variable se deduce de las isotermas registradas, bien porque el área límite disminuye o es independiente de la temperatura, en las monocapas con estructura líquido expandida o líquido condensa$\mathrm{da}$, bien porque con el aumento del valor de la variable, se desplacen hacia zonas del diagrama con menores solicitudes de área, si las monocapas adoptan una estructura gaseosa.

La influencia de la concentración de soluto en la subfase sobre la solubilidad de las moléculas de la monocapa es desigual y función del tipo de soluto. Las citadas dependencias, basadas en los resultados obtenidos con ácido esteárico como constituyente de la monocapa, se resumen en la tabla $V$.

El tipo de soluto que forma parte de la disolución acuosa utilizada como subfase es la variable estudiada que incide sobre la solubilidad de las moléculas de la monocapa con menor nivel de significación. Como pauta de comportamiento general puede establecerse el siguiente orden en el grado de solubilidad de la monocapa, inducido por la subfase:

\section{ETANOL > AGUA > GLICERINA $\approx$ GLUCOSA}

Existen dos casos que presentan dificultades para su inclusión en el orden previamente establecido. Estos son:

* La subfase constituida por la disolución alcohólica con la mayor concentración en etanol ( $2 \mathrm{~mol} / \mathrm{L}$ ) posibilita la disolución prácticamente completa de la monocapa.

* Las monocapas esparcidas sobre sacarosa como soluto de la subfase se caracterizan por poseer una estructura muy expandida que es prácticamente gaseosa incluso a bajas concentraciones de soluto, lo que es posible que potencie la estabilidad de la misma frente a su solubilidad.

Las dependencias entre la composición de la subfase y la temperatura del sistema sobre la estabilidad de las monocapas son de interés en la tecnología de aplicación de los aditivos emulsionantes, ya que una pérdida de tensioactivo por su difusión hacia el seno de la fase provoca una ineficacia de este. Además, pequeñas cantidades de tensioactivos disueltos en la fase continua pueden causar modificaciones en los parámetros físico-químicos de la emulsión de repercusión sobre el proceso. Los resultados obtenidos poseen un interés práctico adicional: estas dependencias pueden permitir el control de la difusión de las moléculas del emulsionante desde el seno de la subfase hacia la monocapa, o viceversa, haciendo que el producto alimentario mantenga sus gotas o burbujas emulsionadas, en todo momento, en las formas más adecuadas para su elaboración, su conservación y su consumo, es decir, optimizando la eficacia del emulsionante no por la inclusión en su molécula de determinados grupos funcionales que modifiquen su afinidad por el medio acuoso, sino por la inclusión en dicho medio de solutos con grupos hidroxilos a una concentración determinada.

\section{BIBLIOGRAFIA}

1. Charalambous, G.; Doxatakis, G., (Ed.).- "Food Emulsifiers: Che mistry, Technology, Functional Properties and Applications". Elsevier, Amsterdam, 1989.

2. Dickinson, E,; Stainsby, G., (Ed.).- "Advances in Food Emulsions and Foams".- Elsevier, Barking (Ingl.), 1988.

3. Krog, N.J.; Riison, T.H.; Larson, K.- "Applications in the Food Industry" in "Encyclopedia of Emulsion Technology".- P. Becher (Ed.). Dekker, New York, 1985.

4. Mc Intyre, R. T.- "Fat-based emulsifiers" in "Fatty Acids in Industry".- R. W. Johnson and E. Fritz (Eds.), Marcel Dekker, Inc., New York, 1989.

5. Boyd, J. V.; Parkinson, C,: Sherman, P.- "Factors affecting emulsion stability and the HLB (hydropholic-lipophilic balance) concept".- J. Colloid Interface Sci. 41 (1972) 359-370.

6. Larsson, K.- "Stability of emulsions formed by polar lipids".- Prog. Chem. Fats Other Lipids 16 (1978) 163-169.

7. Dickinson, E.; Stainsby, G.- "Progress in the formulation of food emulsions and foams".- Food Technol. 41 (9) (1987) 74-81

8. Buhaenko, M. R.; Grundy, M. J.; Richardson, R. M.- "Surface rheology of spread monolayers".- Thin Solid Film. 159 (1988) 171-189.

9. Davies, J. T.; Rideal, E. K.- "Interfacial Phenomena".- Academic Press, 2nd Ed. London, 1963.

10. Lang, H.; Jeschke, P.- "Nonionic Surfactants, Physical Chemistry".- Schick, M. J. (Ed.), Marcel Dekker, Inc. New York, 1987.

11. Multon, J. L. (Ed.).- "Additifs et Auxiliaires de Fabrications dans les Industries Agroalimentaires".- Technique et Documentation Lavoisier, Paris, 1984.

12. Pekalski, A.- "Phase diagrams for a model of a lipid monolayer". - Eur. Biophys. J. 16 (1988) 39-44.

13. Adamson, A. W.- "Physical Chemistry of Surfaces".- John Wiley and Sons., New York, 1976.

14. Rodríguez Patino, J. M.; de la Fuente Feria, J.; Gómez Herrera, C.- "Monocapas de ácidos grasos. I. Acido Esteárico sobre disoluciones acuosas de etanol".- Grasas y Aceites 42 (1991) 114120.

15. Gaines, G.L.-“Insoluble Monolayers at Liquid-Gas Interfaces”.Intercience Publ., New York, 1966.

16. Dickinson, E.- "Effect of atractive forces on monolayer permeation and evaporation resistance".- J. Chem. Soc. Faraday Tran. II. 74 (1978) 821-825.

17. Gershfeld, N.- "The liquid condensed/liquid expanded transition in lipid films: a critical analysis of the film balance experiments".58 (1982) 28-40.

18. Gershfeld, N.- "Physical chemistry of lipids film at fluid interfaces".- Ann. Rev. Phys. Chem. 27 (1976) 349-368.

19. Garti, N.; Sato, K. (Eds.).- "Cristallization and Polymorphism of Fats and Fatty Acids".- Dekker, New York, 1988.

20. Mc Ritchie, F.- "Monolayer compression barrier in emulsion and foam stability".- J. Colloid Interface Sci. 56 (1976) 53-56.

21. Schwartz, P.; Bielecki, J.; Linde, H.- "Origen and behavior of a dissipative structure of the Marangoni-inestability".- Z. Phys. Chem. (Leipzing), 266 (1985) 731-739.

(Recibido: Septiembre 1990) 